\title{
Domain Extensions of Binomial Numbers Applying Successive Sums Transformations on Sequences Indexed by Integers
}

\author{
M. MOESIA B. ${ }^{1 *}$, J. KARAM-FILHO $^{2}$ and G. A. GIRALDI ${ }^{3}$
}

Received on April 3, 2019 / Accepted on November 11, 2019

\begin{abstract}
The classic definition of binomial numbers involves factorials, making unfeasible their extension for negative integers. The methodology applied in this paper allows to establish several new binomial numbers extensions for the integer domain, reproduces to integer arguments those extensions that are proposed in other works, and also verifies the results of the usual binomial numbers.
\end{abstract}

To do this, the impossibility to compute factorials with negative integer arguments is eliminated by the replacement of the classic binomial definition to a new one, based on operations recently proposed and, until now, referred to as transformations by the successive sum applied on sequences indexed by integers. By particularizing these operations for the sequences formed and indexed by integers, it is possible to redefine the usual binomial numbers to any integer arguments, with the advantage that the values are more easily computed by using successive additions instead of multiplications, divisions or possibly more elaborate combinations of these operators, which could demand more than one or two sentences to their application.

Keywords: discrete mathematics, algebraic structures, recursion, sequences, successive product, successive sum, binomial numbers.

\section{INTRODUCTION}

Binomial numbers have an essential role in the most varied fields of pure and applied mathematics and occur in many important algebraic developments. It is possible to cite, among others, the Theory of Numbers and applications in differential and integral calculus, [5]. Normally these

\footnotetext{
*Corresponding author: Marlo Moesia Barroso - https://moesia.org/contact

${ }^{1}$ Coordenação de Métodos Matemáticos e Computacionais, Laboratório Nacional de Computação Científica (LNCC), Av. Getulio Vargas, 333, Quitandinha, 25651-075, Petrópolis, RJ, Brasil. E-mail: moesia.org@gmail.com https://orcid.org/0000-0002-6093-3828

${ }^{2}$ Coordenação de Modelagem Computacional, Laboratório Nacional de Computação Científica (LNCC), Av. Getulio Vargas, 333, Quitandinha, 25651-075, Petrópolis, RJ, Brasil. E-mail: jkfi@ lncc.br https://orcid.org/0000-0002-1952-3202

${ }^{3}$ Coordenação de Métodos Matemáticos e Computacionais, Laboratório Nacional de Computação Científica (LNCC), Av. Getulio Vargas, 333, Quitandinha, 25651-075, Petrópolis, RJ, Brasil. E-mail: gilson@lncc.br https://orcid.org/0000-0003-0623-9461
} 
constants appear when the operations contained in $(a+b)^{n}$, being $n$ a natural power, are developed and the various terms grouped according to the $\mathrm{a}$ and $\mathrm{b}$ resulting exponents. However, other compound operations without the aspect of potentiation can produce them, as will be seen in this article.

The earliest known binomial application example is due to Euclid (325 BC-265 BC) which through geometry concluded that the square area is equal to the sum of the rectangles areas contained in it [2], whereas the representation of binomial constants in the form of a table is usually attributed to the Chinese mathematician Yang Hui (1238 AD-1298 AD), although there are some divergences between references. Posteriorly, the binomial numbers were also studied by Blaise Pascal (1623 AD-1662 AD) and more recently by Newton, Euler, Abel, and Gauss among others [11].

The usual binomial numbers are defined by a combination of products and divisions involving the natural numbers in factorial forms. In turn, these factorials were extended to real arguments through the gamma function by Euler [1], allowing to establish an infinity of binomial results in $\mathbb{R}$. Nevertheless, the domain limitation of the usual binomials has remained, because the gamma function is not defined for negative integer arguments. In recent decades, efforts have been made to overcome this difficulty, usually by extending some property to the set $\mathbb{Z}^{-}$of the usual binomial numbers, as done in [4] and [8].

This paper presents in a synthetic form how to define and extend the usual binomial numbers for any integer arguments, according to the proposition made in [9], which changed the approach to the question. It deepens the brief exposition presented in [10]. For this purpose, it was not used factorials or some kind of product in the definition of binomials; instead, the so-denominated transformations by the successive sum on sequences indexed by integer numbers defined applying additions have been used. Since such transformations can be performed within any arguments in $\mathbb{Z}$, it has become possible to obtain binomial numbers easily, both in the usual natural domain and in the integer one. These results are consistent with those of other authors cited and are obtained considering a set of interest properties that must remain valid to any extension.

Behind this proposition there is a new methodology for approaching recursive functions, through mathematical operations that transform integer-indexed sequences from the solution of a system of equations composed of a known value and a recursion. For transformation orders greater or equal than two and strictly positive arguments, it is verified that the solution obtained for this system can be computed by nested summations. But, as it will be seen in this work, nested summations had not been used in the cases of: unit and null orders, and negative first order. 


\section{SUCCESSIVE SUM APPLIED ON SEQUENCES INDEXED BY INTEGER NUMBERS}

The successive sums introduced in [9] can be defined as follows.

\section{Definition 2.1.}

Let $G(A,+)$ be a group, where $A$ is any non-empty set, and $S Z$ the set of all sequences in $A$ indexed by integers, with the general form

$$
\left(\alpha_{i}\right)_{i \in \mathbb{Z}}=\left(\ldots, \alpha_{-2}, \alpha_{-1}, \alpha_{0}, \alpha_{1}, \alpha_{2}, \ldots, \alpha_{i}, \ldots\right)
$$

then, with these conditions, it was recursively defined the function $S_{\mathbb{Z}}: S Z \times \mathbb{Z} \rightarrow A$ by

$$
\left\{\begin{array}{l}
S_{\mathbb{Z}}\left(\left(\alpha_{i}\right), 0\right)=\eta_{(+)}, \text {and } \\
S_{\mathbb{Z}}\left(\left(\alpha_{i}\right), z+1\right)=S_{\mathbb{Z}}\left(\left(\alpha_{i}\right), z\right)+\alpha_{z+1}, \forall z \in \mathbb{Z},
\end{array}\right.
$$

called "successive sum of $\left(\alpha_{i}\right)$, calculated in $i=z$ ", where $\eta_{(+)}$is the neutral element of the binary operation $(+)$.

For convenience, $S_{\mathbb{Z}}\left(\left(\alpha_{i}\right), z\right)$ will be symbolized by the notation

$$
\mathcal{S}_{\mathbb{Z}}\left(\left(\alpha_{i}\right), z\right)=\alpha_{i+}^{\mid i=z} ; \forall z \in \mathbb{Z} .
$$

It should be noted that according to the symbology adopted for successive operations, the binary operator + can be any one applicable on the terms of the sequence $\left(\alpha_{i}\right)$ but, as well as associative, it must also be commutative.

The values of the successive sum for negative $z$ are computed by the same expressions (2.2)(2.3), but using the recursion as a system of equations and doing $z=-1,-2,-3$, and so on. When considering all integers, it is possible to obtain the following expressions by finite induction:

$$
\begin{aligned}
& \alpha_{i+}^{\mid i=z}=\alpha_{1}+\alpha_{2}+\alpha_{3}+\ldots+\alpha_{z-1}+\alpha_{z} ; \forall z \in \mathbb{Z}^{*+}, \\
& \alpha_{i+}{ }^{\mid i=0}=\eta_{(+)}, \text {and } \\
& \alpha_{i+}{ }_{i=z}=\left(-\alpha_{0}\right)+\left(-\alpha_{-1}\right)+\left(-\alpha_{-2}\right)+\ldots+\left(-\alpha_{z+2}\right)+\left(-\alpha_{z+1}\right) ; \forall z \in \mathbb{Z}^{-},
\end{aligned}
$$

where the symbol - placed before each $\alpha_{i}$ indicates the symmetric of the respective term. When attempting to write expressions (2.5)-(2.7) in the form of a single summation one realizes that it is not possible. On the other hand, any summation can be written as a successive sum:

$$
\alpha_{i+m-1} \mid i=n-m+1=\sum_{i=m}^{n} \alpha_{i} ; \forall m \leqslant n, m \text { and } n \in \mathbb{Z} .
$$

It should be noted that all summation can be represented by a successive sum, but it is impossible to keep the positive step +1 and make the upper index smaller than the lower one. In addition, all results in (2.8) are computed using only the expression (2.5), what clarifies why it was initially 
stated that successive sums are more generic than summations. Another aspect is the importance of keeping the variable $i$ explicit, to allow making assignments to it. In (2.8) these assignments have been done in two different ways: one in terms of the sequence index and the other in terms of the value to be computed. In the sequence's terms $\alpha_{i}$ the assignment $i \leftarrow i+m-1$ produces the range $\left\{\alpha_{m}, \alpha_{n}\right\}$ of values to be computed, while making $i=n-m+1$ results in the quantity $m-n+1$ of these values.

\section{TRANSFORMATIONS OF SEQUENCE BY THE SUCCESSIVE SUM}

In this paper the new sequence created from the results of a successive operation will be called transformation, then:

\section{Definition 3.2.}

The integer sequence transformation by the successive sum $T_{S z}: S Z \rightarrow S Z$ is defined as the sequence formed by all values of a given successive sum calculated in $i=z$ :

$$
\left(\alpha_{i_{+}}^{\backslash i=z}\right)_{z \in \mathbb{Z}}=\left(\ldots, \alpha_{i_{+}}^{\mid i=-1}, \alpha_{i_{+}}^{\mid i=o}, \alpha_{i_{+}}^{\mid i=1}, \ldots, \alpha_{i+}^{\mid i=z}, \ldots\right) .
$$

The notations introduced in [9] will also be used here:

$$
\left(\alpha_{i+}^{\mid i=z}\right)_{z \in \mathbb{Z}}=\alpha_{i_{+}}{ }^{i}=\alpha_{i+}{ }^{i} .
$$

The new sequence $T_{\mathrm{Sz}}$ can also be transformed, producing another $T_{\mathrm{Sz}}$ :

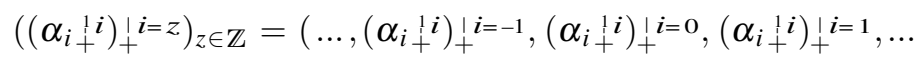

$$
\begin{aligned}
& \left.\ldots,\left(\alpha_{i+}{ }^{1} i\right)_{+}^{\lfloor} i=z, \ldots\right) \text {. }
\end{aligned}
$$

By analogy to the symbolism of (3.2), it has the following notations:

$$
\left(\left(\alpha_{i+}{ }^{i}{ }^{i}\right)_{+}^{\lfloor i=z}\right)_{z \in \mathbb{Z}}=\left(\alpha_{i+}{ }^{1}{ }^{i}\right)_{+}^{\lfloor}{ }^{i}=\alpha_{i+}^{2}{ }^{i} .
$$

so that (3.3) can be represented more compactly as

$$
\left(\alpha_{i+}^{2} i=z\right)_{z \in \mathbb{Z}}=\left(\ldots, \alpha_{i+}^{2} i=-1, \alpha_{i+}^{2} i=0, \alpha_{i+}^{2} i=1, \ldots, \alpha_{i+}^{2} i=z, \ldots\right),
$$

which is the second order integer sequence transformation by the successive sum.

Naturally, this process can be repeated without great difficulty indefinitely. In the following expression we have the $T_{\mathrm{Sz}}$ of order $n+1$, from the $T_{\mathrm{Sz}}$ of order $n$ :

$$
\left(\left(\alpha_{i+}^{n}{ }^{i}\right)_{+}^{\lfloor} i=z\right)_{z \in \mathbb{Z}}=\left(\alpha_{i+}^{n} i\right)_{+}^{i}=\alpha_{i}^{n+1} i ; n \in \mathbb{N}^{*} .
$$

Similarly to what was done to extend the successive sums to negative integer values, the integer sequence transformations may have their orders extended to non strictly positive integer arguments. The construction of $T_{\mathrm{Sz}}$ for any negative order also applies the dualistic interpretation 
of the recursions in these transformations and is more detailed in [9]. Thus, the null order integer sequence transformation by the successive sum is obtained assuming that it is possible to consider $n=0$ in (3.6):

$$
\left(\left(\alpha_{i+}^{0}{ }^{i}\right)_{+}^{\lfloor i=z}\right)_{z \in \mathbb{Z}}=\left(\alpha_{i+}^{0} i\right)_{+}^{\lfloor} i=\alpha_{i+}{ }^{i}
$$

By interpreting (3.7) as an equation, one can determine which are the terms of the sequence $\alpha_{i}{ }_{i}^{i}$ such that its first-order transformation produces (3.1), resulting in

$$
\left(\alpha_{i+}^{0} i=z\right)_{z \in \mathbb{Z}}=\left(\ldots, \alpha_{-2}, \alpha_{-1}, \alpha_{0}, \alpha_{1}, \alpha_{2}, \ldots, \alpha_{z}, \ldots\right),
$$

therefore the null-order transformation is the identity of those transformations.

Likewise the previous case, it is possible to admit $n=-1$ in (3.6):

$$
\left(\left(\alpha_{i+}^{-1} i\right)_{+}^{\lfloor i=z}\right)_{z \in \mathbb{Z}}=\left(\alpha_{i+}^{-1} i\right)_{+}^{\lfloor i}=\alpha_{i+}^{0} i .
$$

For a new extension, (3.9) is interpreted again as an equation, which results in the first negative order integer sequence transformation by the successive sum:

$$
\begin{gathered}
\left(\alpha_{i+1}^{-1} i=z\right)_{z \in \mathbb{Z}}=\left(\ldots,-\alpha_{-2}+\alpha_{-1},-\alpha_{-1}+\alpha_{0},-\alpha_{0}+\alpha_{1},-\alpha_{1}+\alpha_{2}, \ldots\right. \\
\left.\ldots,-\alpha_{z-1}+\alpha_{z}, \ldots\right),
\end{gathered}
$$

where $-\alpha$ is the symmetrical element of $\alpha$. This process can be repeated indefinitely for all negative orders.

It is noted that the integer-sequence transforms by the successive sum also have an inverse transform, which is different from (3.10) with respect to the subset of sequences in which it can be applied. This type of difference occurs for all applications of inverse transformations of the transform $T_{\mathrm{Sz}}$, that is: the second, third, fourth, and the subsequent inverse transformations.

The recursive property relating the transforms $T_{\mathrm{Sz}}$ of order $k$ and $k+1$ to any integer arguments is given by the expression (see [9], items 5.1.5 and 5.1.6):

$$
\alpha_{i}^{k+1} i=z+1=\alpha_{i}{ }^{k+1} i=z+\alpha_{i}{ }^{k} i=z+1 ; \forall k \text { and } z \in \mathbb{Z} .
$$

The transformations $T_{\mathrm{Sz}}$ have among their properties some that include the usual binomial numbers. For example, using (3.11) it is possible to determine the expressions for the general term of the sequence of the various negative order transforms, part of which is shown in Figure 1.

The natural constants that can be observed from the columns in Figure 1 are the values of the table of Yang Hui, cited in the introduction of this article, that can be arranged in the form of a triangular table, called Pascal triangle or triangle of Tartáglia. Besides of being easily obtained by the addition algorithm highlighted in Figure 1 (see the construction of columns $\mathrm{C}_{1}-\mathrm{C}_{4}$ ), it is also known that they correspond to the usual binomial numbers, although no potentiation is being used here. As will be seen, from the advent of the transformations $T_{S z}$, there are also new alternatives to represent these constants. In fact, let $(i)$ be the usual sequence of integers, that is:

$$
(i)_{i \in \mathbb{Z}}=(\ldots,-2,-1,0,1,2, \ldots, i, \ldots) .
$$




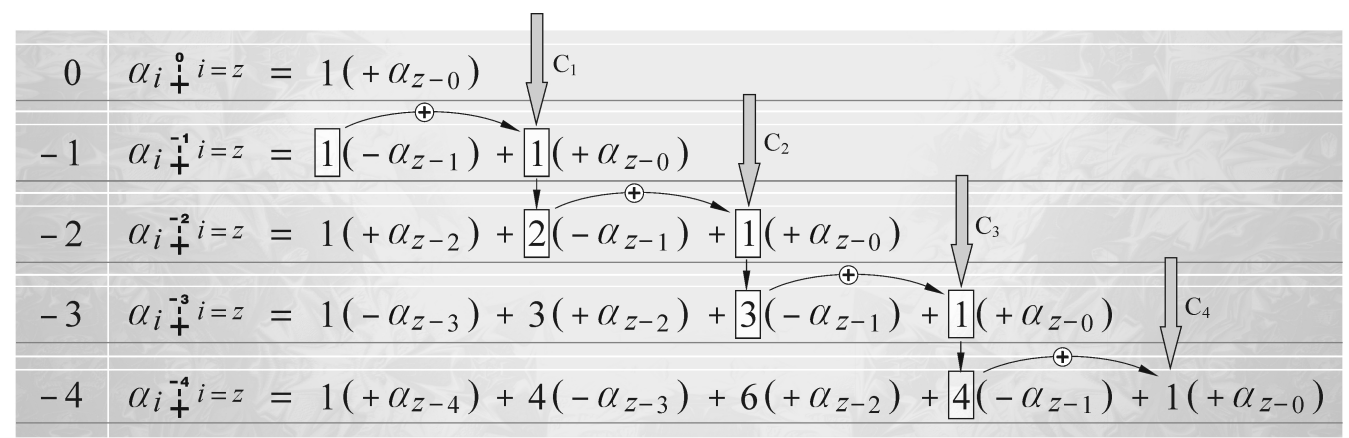

Figure 1: Examples of the generic term of the sequences $\left(\alpha_{i}\right)$ resulting from $T_{\mathrm{Sz}}$ transforms of negative order.

By applying (3.10) on the sequence $(i)$, it follows that

$$
i_{+}^{-1} i=z=-(z-1)+z=1 ; \forall z \in \mathbb{Z} .
$$

The same can be done by applying (3.8) on the sequence $(i)$ :

$$
i_{+}^{0} i=z=z ; \forall z \in \mathbb{Z} \text {. }
$$

Following for orders 1, 2, 3, etc., all constants of Figure 1 can be obtained.

In order to simplify the symbology of $T_{\mathrm{Sz}}$ applied to the sequence $(i)$, that is, the sequence of the integers themselves, the following notation has been established:

$$
i_{+}^{k} i=z=z_{+}^{k} ; \forall k \text { and } z \in \mathbb{Z} .
$$

With the same notation as in (3.15) the values that compose the triangle of Pascal, and therefore the constants of Figure 1, can be represented according to Figure 2.

\section{FIRST EXTENSION OF BINOMIAL NUMBERS}

In this section, using a new approach it will be verified how it is possible to apply the transformations by the successive sum to represent the binomial numbers by additions, as well as to extend them to any integer arguments. To do that, it will be necessary the following result, Corollary 4.0 below, here obtained by a different demonstration than the usual one, [3], which considers nested summations.

\section{Corollary 4.0 (Binomials by the Successive Sum).}

Any usual binomial number can be calculated through transformations by the successive sum on the natural numbers sequence applying the expression

$$
\left(\begin{array}{l}
n \\
k
\end{array}\right)=i_{+}^{k-1} i=n-k+1 ;(n-k) \geqslant 0 ; \forall k \text { and } n \in \mathbb{N} .
$$




\begin{tabular}{|c|c|}
\hline 0 & $\alpha_{i} \stackrel{0}{+}^{i=z}=1 \ddot{\mathfrak{t}}^{-1}\left(+\alpha_{z-0}\right)$ \\
\hline-1 & 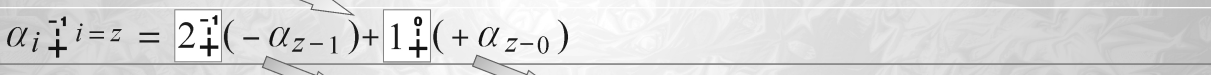 \\
\hline-2 & $\alpha_{i} \ddot{+}^{-2} i=z=3 \vec{i}\left(+\alpha_{z-2}\right)+2 \grave{\dot{t}}\left(-\alpha_{z-1}\right)+1 \dot{t}\left(+\alpha_{z-0}\right)$ \\
\hline-3 & 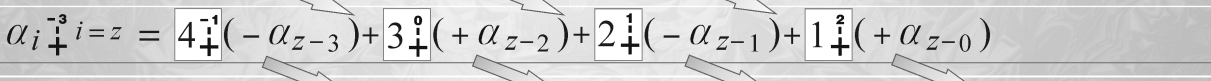 \\
\hline-4 & 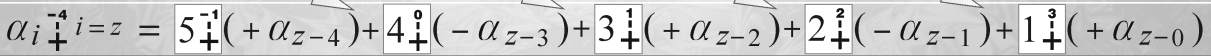 \\
\hline
\end{tabular}

Figure 2: Examples of the generic term of the sequences $\left(\alpha_{i}\right)$ resulting from the negative order transform $T_{\mathrm{Sz}}$, with the constants represented by transformations of the natural numbers by the successive sum.

\section{Proof.}

Let $(z+i)$ be the sequence defined by

$$
(z+i)_{i \in \mathbb{Z}}=(\ldots, z-2, z-1, z+0, z+1, z+2, \ldots, z+i, \ldots) ; z \in \mathbb{Z} .
$$

A significant property of the transformations by the successive sum, demonstrated in [9] (chapter 6, item 6.1.2), establishes that

$$
i_{+}^{n} i=z=\frac{z(z+i) !{ }^{i=n}}{(n+1) !} ; \forall n \in \mathbb{N} \text { and } \forall z \in \mathbb{Z},
$$

where $(z+i) !^{i=n}$ is the successive product of the $(z+i)$ sequence, calculated in $i=n$, and $(n+$ $1)$ ! is the usual factorial given by $(n+1)(n)(n-1) \ldots(2)(1)$. Since the usual multiplication "." is associative and commutative, it can be considered as a " + " operator, then (2.2)-(2.3) can be applied, but in this case the neutral element is 1 .

According to (4.3) any product of successive integers can be done through $i_{+}^{n} i=z$, so it is expected that it also represents a quotient of factorials. Indeed, firstly, the domain of (4.3) can be extended, allowing $\mathrm{n}=-1$. After that, doing the assignment $n \leftarrow k-1, k \in \mathbb{N}$, one has

$$
i_{+}^{k-1} i=z=\frac{z(z+i) !^{i=k-1}}{k !} ; \forall k \in \mathbb{N} \text { and } \forall z \in \mathbb{Z} .
$$

Now, by making the assignment $z \leftarrow n-k+1,(n-k) \geqslant 0$ in (4.4):

$$
\stackrel{i_{+}^{k-1}}{i=n-k+1}=\frac{(n-k+1)(n-k+1+i) !^{i=k-1}}{k !} ;(n-k) \geqslant 0 ; k, n \in \mathbb{N} .
$$


The equality (4.5) can also be expressed by the factorial quotient that defines binomial numbers. There are three cases to be checked: $k>1, k=1$ and $k=0$. For the first one, opening the indicated operations in the numerator, one has

$$
\begin{aligned}
i_{+}^{k-1}+i=n-k+1 & =\frac{(n-k+1)[(n)(n-1)(n-2) \ldots(n-k+3)(n-k+2)]}{k !}= \\
& =\frac{n !}{(n-k) ! k !}=\left(\begin{array}{l}
n \\
k
\end{array}\right) ; k>1,(n-k) \geqslant 0 ; k \text { and } n \in \mathbb{N} .
\end{aligned}
$$

The two remaining cases can be easily verified (see [9], item 6.2.3). Then it is demonstrated (4.1).

Figure 3 confirms (4.1) through some examples.

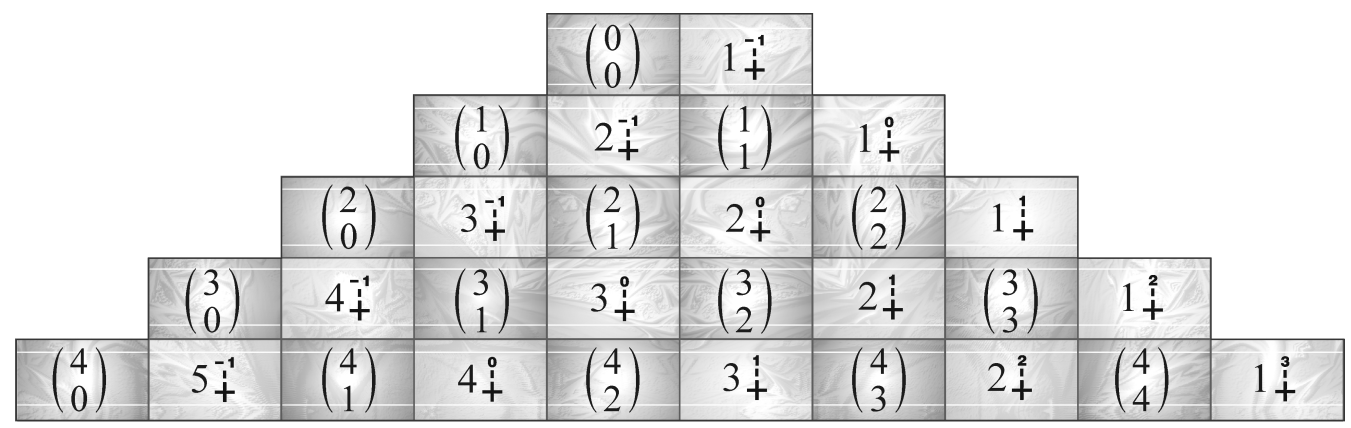

Figure 3: Binomial numbers arranged in the Pascal Triangle, represented by usual symbology and followed by their respective $T_{S z}$ transformation in the compact form: $i^{k-1}{ }^{i=n-k+1}=(n-k+1)^{k-1} \stackrel{+}{+}$.

As highlighted in the introduction, no extension by the gamma function is defined for negative integer arguments, that is: there is no result for the factorials of $-1,-2,-3$, etc. On the other hand, the second member of (4.1) is only composed of additions and can be calculated for any integer arguments, so the first extension that can be proposed for binomial numbers is simply to apply the successive sum of (4.1) also to negative values of $n$ and $k$ :

\section{Binomial Extension 1.}

Let $G(\mathbb{Z},+)$ be the usual group of integers and $(i)$ the integer's sequence, with elements in the general form given in (3.12). Under these conditions the binomial number $n$ choose $k$, or the combinatorial number $n$ choose $k$, is defined by

$$
\left(\begin{array}{l}
n \\
k
\end{array}\right)=i_{+}^{k-1} i=n-k+1 ; \forall k \text { and } n \in \mathbb{Z}
$$

Part of the new values for binomial numbers that can be computed using (4.7) are null, what will be demonstrated in what follows. 
Property 1. First Extension Binomials with Null Values:

$$
\left(\begin{array}{l}
n \\
k
\end{array}\right)=0 ; \forall k \leqslant-1 \text { and } n \in \mathbb{Z}
$$

Proof.

Reordering (3.11) and considering as its particular case the sequence (3.12):

$$
i_{+}^{k} i=z+1=i_{+}^{k+1} i=z+1-i_{+}^{k+1} i=z ; \forall k \text { and } z \in \mathbb{Z} .
$$

If $k=-2$ it is possible to apply (3.13) into the second member of (4.9):

$$
i_{+}^{-2} i=z+1=i_{+}^{-1} i=z+1-i_{+}^{-1} i=z=1-1=0 ; \forall z \in \mathbb{Z} .
$$

By the reusing of (4.9) with the result obtained in (4.10) and so on, it is possible to get null results for all other negative values of $k$ :

$$
i_{+}^{k} i=z=0 ; \forall k \leqslant-2 \text { and } z \in \mathbb{Z} .
$$

From (4.11), it is clear that there exists one subset with only null results in (4.7). To confirm the subset $\forall k \leqslant-1$ and $z \in \mathbb{Z}$, specified in (4.8), consider the assignments $z \leftarrow n-k+1$ and $k \leftarrow k-1$ in (4.11).

When considering binomial numbers with $k>-1$ and $n-k<0$ the extension has results that are not necessarily null. For each $k$ there is some expression easily inducible (see (3.13)), as

$$
\left(\begin{array}{l}
n \\
0
\end{array}\right)=1 ; \forall n \in \mathbb{Z}^{-},
$$

or it can also be obtained using (4.5) with its domain extended:

$$
\left(\begin{array}{l}
n \\
k
\end{array}\right)=\frac{(n-k+1)(n-k+1+i) !^{i=k-1}}{k !} ; k \geqslant 0,(n-k)<0 .
$$

Figure 4 exemplifies some values of this extension.

What prevents to consider expression (4.7) as the definitive extension for the binomial numbers is the fact that it is not the only one possible, as will be seen in the next section. Moreover, it was not demonstrated that this is the best extension for any application or, at least, for most of them. These doubts lead to more embracing studies evolving successive sums as a tool for extending binomial numbers, as proposed in the following.

\section{BINOMIAL NUMBERS ON ANY SEQUENCE THROUGH SEQUENCE'S TRANSFORMATIONS BY THE SUCCESSIVE SUM}

It is easy to verify that the simple change of the sequence $(i)$ by other $\left(\alpha_{i}\right)$ allows to obtain an infinity of different results through integer sequence transformations, so that it comes out the 


\begin{tabular}{|c|c|c|c|c|c|c|c|c|c|}
\hline$-1 \tilde{t}^{-4}$ & 0 & $\left(\begin{array}{l}-5 \\
-2\end{array}\right)$ & $-2_{i}^{-3}$ & 0 & $\left(\begin{array}{l}-5 \\
-1\end{array}\right)$ & $-3 i_{i}^{-2}$ & 0 & $\left(\begin{array}{r}-5 \\
0\end{array}\right)$ & $-4 i_{i}^{-1}$ \\
\hline 0 & $\left(\begin{array}{l}-4 \\
-2\end{array}\right)$ & $-11_{+}^{-3}$ & 0 & $\left(\begin{array}{l}-4 \\
-1\end{array}\right)$ & $-2 \ddot{t}^{-2}$ & \multicolumn{2}{|c|}{ First Extension } & $-3 \ddot{t}^{-1}$ & 1 \\
\hline$\left(\begin{array}{l}-3 \\
-2\end{array}\right)$ & $0 \stackrel{-3}{+}$ & 0 & $\left(\begin{array}{l}-3 \\
-1\end{array}\right)$ & $-11_{+}^{-2}$ & 0 & $\left(\begin{array}{r}-3 \\
0\end{array}\right)$ & $-2 \ddot{t}^{-1}$ & 1 & $\left(\begin{array}{r}-3 \\
1\end{array}\right)$ \\
\hline $1_{+}^{-3}$ & 0 & $\left(\begin{array}{l}-2 \\
-1\end{array}\right)$ & $0 \ddot{+}_{+}^{-2}$ & 0 & $\left(\begin{array}{r}-2 \\
0\end{array}\right)$ & $-1{ }^{-1}$ & 1 & $\left(\begin{array}{r}-2 \\
1\end{array}\right)$ & $-2 \stackrel{0}{+}$ \\
\hline 0 & $\left(\begin{array}{l}-1 \\
-1\end{array}\right)$ & $1 \dddot{t}^{2}$ & 0 & $\left(\begin{array}{r}-1 \\
0\end{array}\right)$ & $0 \bar{t}^{-1}$ & 1 & $\left(\begin{array}{r}-1 \\
1\end{array}\right)$ & $-1 \stackrel{0}{+}$ & -1 \\
\hline$\left(\begin{array}{r}0 \\
-1\end{array}\right)$ & $2{ }_{+}^{-2}$ & 0 & $\left(\begin{array}{l}0 \\
0\end{array}\right)$ & $1 \ddot{t}$ & 1 & $\left(\begin{array}{l}0 \\
1\end{array}\right)$ & $0 \stackrel{\circ}{+}$ & 0 & $\left(\begin{array}{l}0 \\
2\end{array}\right)$ \\
\hline $3 \ddot{+}_{+}^{-2}$ & 0 & $\left(\begin{array}{l}1 \\
0\end{array}\right)$ & $2 \vec{t}$ & 1 & $\left(\begin{array}{l}1 \\
1\end{array}\right)$ & $1 \stackrel{0}{+}$ & 1 & $\left(\begin{array}{l}1 \\
2\end{array}\right)$ & $0+$ \\
\hline 0 & $\left(\begin{array}{l}2 \\
0\end{array}\right)$ & $3 \ddot{t}^{-1}$ & 1 & $\left(\begin{array}{l}2 \\
1\end{array}\right)$ & $2 \stackrel{0}{+}$ & 2 & $\left(\begin{array}{l}2 \\
2\end{array}\right)$ & $1+$ & 1 \\
\hline$\left(\begin{array}{l}3 \\
0\end{array}\right)$ & $4{ }_{i}^{-1}$ & 1 & Orig & al Bin & iial Nu & abers & 24 & 3 & $\left(\begin{array}{l}3 \\
3\end{array}\right)$ \\
\hline $5 \bar{t}^{-1}$ & 1 & $\left(\begin{array}{l}4 \\
1\end{array}\right)$ & $4 \stackrel{\leftrightarrow}{+}$ & 4 & $\left(\begin{array}{l}4 \\
2\end{array}\right)$ & 31 & 6 & $\left(\begin{array}{l}4 \\
3\end{array}\right)$ & $2 \stackrel{2}{+}$ \\
\hline 1 & $\left(\begin{array}{l}5 \\
1\end{array}\right)$ & $5 \stackrel{+}{+}$ & 5 & $\left(\begin{array}{l}5 \\
2\end{array}\right)$ & $4+$ & 10 & $\left(\begin{array}{l}5 \\
3\end{array}\right)$ & $3 \stackrel{2}{+}$ & 10 \\
\hline
\end{tabular}

Figure 4: The first extension of the binomial numbers, according to (4.7). The darkest area corresponds to (4.8), in the half-light it is the traditional Pascal triangle, and in the illuminated area there are some extended results, given by (4.12) and (4.13).

question on how to find which sequence or sequences are appropriate to obtain the binomial numbers of interest. It is also possible to study the question in a more general sense by considering any sequences indexed by integers and verifying what properties of the usual binomial numbers are preserved.

In the following, it is presented the binomial numbers definition on any sequences.

\section{Definition 5.3.}

Let $G(A,+)$ be a group and $S Z$ the set of integer sequences on $A$, with elements in the general form given in (2.1). Under these conditions the binomial number $n$ choose $k$ on sequence $\left(\alpha_{i}\right)$, or the combinatorial number $n$ choose $k$ on sequence $\left(\alpha_{i}\right)$, is defined by

$$
{ }_{n} C\left(\alpha_{i}\right)_{k}=\left(\begin{array}{l}
n \\
k
\end{array}\right)_{\alpha_{i}}=\alpha_{i}{ }^{k-1} \dot{+} i=n-k+1 ; \forall k \text { and } n \in \mathbb{Z} .
$$

Considering the sequence of integers themselves the expression (5.1) becomes equal to the expression (4.7), represented as ${ }_{n} \mathrm{C}(i)_{k}$, then being a generalization made from the first extension of binomial numbers which was proposed in the previous topic. 


\subsection{Stifel's Relation for Binomial Numbers on any Integer Sequence}

A remarkable additive relation due to Definition 5.3 is

Property 2. Generalized Stifel's Relation:

$$
\left(\begin{array}{l}
n+1 \\
k+1
\end{array}\right)_{\alpha_{i}}=\left(\begin{array}{c}
n \\
k+1
\end{array}\right)_{\alpha_{i}}+\left(\begin{array}{l}
n \\
k
\end{array}\right)_{\alpha_{i}} ; \forall k \text { and } n \in \mathbb{Z}
$$

\section{Proof.}

The main recursive property of the transformations $T_{\mathrm{Sz}}$, given by (3.11), is

$$
\alpha_{i}^{k+1} i=z+1=\alpha_{i}^{k+1} i=z+\alpha_{i}{ }_{+}^{k} i=z+1 ; \forall k \text { and } z \in \mathbb{Z} .
$$

Doing the assignment $k \longleftarrow k-1$ in expression (5.3), it becomes

$$
\alpha_{i+\dot{+}}{ }^{i=z+1}=\alpha_{i+}^{k}{ }^{i=z}+\alpha_{i}{ }_{+}^{k-1} i=z+1 ; \forall k \text { and } z \in \mathbb{Z} \text {. }
$$

Now, doing the assignment $z \longleftarrow n-k$ in expression (5.4), it leads to:

$$
\alpha_{i+\dot{+}}{ }^{k} i=n-k+1=\alpha_{i+\dot{+}}^{k} i=n-k+\alpha_{i}{ }^{k-1} i=n-k+1 ; \forall k \text { and } n \in \mathbb{Z} \text {. }
$$

Each term in (5.5) can be interpreted as a binomial number on the sequence $\left(\alpha_{i}\right)$, according to the following expressions:

$$
\begin{aligned}
& \alpha_{i+\dot{+}}{ }^{k} i=n-k+1=\alpha_{i+\dot{+}}{ }^{k} i=(n+1)-(k+1)+1={ }_{n+1} \mathrm{C}\left(\alpha_{i}\right)_{k+1} ; \forall k \text { and } n \in \mathbb{Z}, \\
& \alpha_{i \dot{+}}{ }^{k}{ }^{i=n-k}=\alpha_{i \dot{+}}{ }^{i=n-(k+1)+1}={ }_{n} \mathrm{C}\left(\alpha_{i}\right)_{k+1} ; \forall k \text { and } n \in \mathbb{Z}, \\
& \alpha_{i}^{k-1} i=n-k+1={ }_{n} \mathrm{C}\left(\alpha_{i}\right)_{k} ; \forall k \text { and } n \in \mathbb{Z} \text {. }
\end{aligned}
$$

Therefore (5.5) can be expressed in terms of binomial numbers on the sequence $\left(\alpha_{i}\right)$ substituting each term according to (5.6)-(5.8), that is:

$$
{ }_{n+1} \mathrm{C}\left(\alpha_{i}\right)_{k+1}={ }_{n} \mathrm{C}\left(\alpha_{i}\right)_{k+1}+{ }_{n} \mathrm{C}\left(\alpha_{i}\right) k ; \forall k \text { and } n \in \mathbb{Z} .
$$

Changing the notation, expressions (5.9) and (5.2) are equal.

It is observed that (5.2) is the generalization for any integer sequence $\left(\alpha_{i}\right)$ of the well known Stifel relation of usual binomial numbers [2]. Note that the property is valid even for the extension of binomials with negative arguments.

The consistency of Definition 5.3 with respect to the Stifel relation suggests that this generalization may preserve other properties of the usual binomial numbers. 


\subsection{Other Properties for Binomials on Integers Sequences}

Independently of sequence $\left(\alpha_{i}\right)$ some results of ${ }_{n} \mathrm{C}_{(\alpha i) k}$ can be easily predicted as a result of properties of the transforms $T_{\mathrm{Sz}}$.

Property 3. Binomial's Value on Integers Sequences if $k=0$ :

$$
\left(\begin{array}{l}
z \\
0
\end{array}\right)_{\alpha_{i}}=-\alpha_{z}+\alpha_{z+1} ; \forall z \in \mathbb{Z} \text { and } \forall\left(\alpha_{i}\right) \in S Z
$$

\section{Proof.}

If the order is -1 , then for any sequence it follows that the result of the general term of the sequence of $T_{\mathrm{Sz}}$ in (3.10) is given by

$$
\alpha_{i}^{-1} i=z=-\alpha_{z-1}+\alpha_{z} ; \forall\left(\alpha_{i}\right) \in S Z .
$$

Doing the assignment $z \longleftarrow z+1$ in expression (5.11), it becomes

$$
\alpha_{i+}^{-1} i=z+1=-\alpha_{z}+\alpha_{z+1} ; \forall\left(\alpha_{i}\right) \in S Z
$$

Thus, considering $n \longleftarrow z$ in the definition (5.1), it can be stated that

$$
{ }_{z} \mathrm{C}\left(\alpha_{i}\right)_{0}=\alpha_{i+}^{-1}{ }_{i=z+1}^{1} ; \forall z \in \mathbb{Z} .
$$

Using the usual binomial notation, (5.12) and (5.13), it leads to (5.10).

From expression (5.10), it is possible to inquire which sequences $\left(\alpha_{i}\right)$ preserve the remarkable value of the usual binomial numbers given by

$$
\left(\begin{array}{l}
n \\
0
\end{array}\right)=\frac{n !}{(n-0) ! 0 !}=1 ; \forall n \in \mathbb{N} .
$$

For example, by restricting to the real numbers, any sequence in the form

$$
\left(\beta_{i}(x)\right)_{i \in \mathbb{Z}}=(\ldots, x-2, x-1, x+0, x+1, x+2, \ldots, x+i, \ldots) ; \forall x \in \mathbb{R}
$$

satisfies $-\beta_{z}(x)+\beta_{z+1}(x)=-\alpha_{z}+\alpha_{z+1}=1$, then

$$
\left(\begin{array}{l}
z \\
0
\end{array}\right)_{\beta_{i}(x)}=1 ; \forall z \in \mathbb{Z} \text { and } \forall\left(\beta_{i}(x)\right) \in S Z .
$$

Property 4. Binomial's Value on Integers Sequences if $k=n$ :

$$
{ }_{n} C\left(\alpha_{i}\right)_{n}=\left(\begin{array}{l}
n \\
n
\end{array}\right)_{\alpha_{i}}=\alpha_{1} ; \forall n \in \mathbb{Z}^{*+} .
$$




\section{Proof.}

Applying the definition (5.1) in this case, it follows that

$$
{ }_{n} \mathrm{C}\left(\alpha_{i}\right)_{n}=\left(\begin{array}{l}
n \\
n
\end{array}\right)_{\alpha_{i}}=\alpha_{i}^{n-1} i=1 ; \forall n \in \mathbb{Z} .
$$

Using the recursive property (3.11) of $T_{\mathrm{Sz}}$, for the case $z=0$ :

$$
\alpha_{i}^{k+1} i=1=\alpha_{i}^{k+1} i=0+\alpha_{i}^{k}{ }_{+}^{k} i=1 ; \forall k \in \mathbb{Z} .
$$

According to [9] and by definition, any $T_{\mathrm{Sz}}$ calculated at zero results in the neutral element of + for any strictly positive integer order, therefore

$$
\begin{aligned}
& \alpha_{i}{ }_{+}^{k+1} i=1=\eta_{(+)}+\alpha_{i+\stackrel{*}{k}} i=1 ; \forall k \in \mathbb{Z}^{+}, \\
& \alpha_{i}{ }^{k+1} i=1=\alpha_{i}{ }^{k}{ }^{i=1} ; \forall k \in \mathbb{Z}^{+} .
\end{aligned}
$$

Using (3.8) or considering that the null order does not perform any transformation on $\left(\alpha_{i}\right)$, it follows that

$$
\alpha_{i} \stackrel{0}{i=1}=\alpha_{1} .
$$

Substituting (5.22) into (5.21) for $k=0,1,2,3$ and so on, it can be demonstrated by finite induction that the result is the same for all other orders $k$ :

$$
\alpha_{i \stackrel{+}{k}}{ }^{i=1}=\alpha_{1} ; \forall k \in \mathbb{Z}^{+} .
$$

Returning to (5.18) with the result (5.23) it is obtained (5.17).

Another remarkable value of the usual binomial numbers is calculated by

$$
\left(\begin{array}{l}
n \\
n
\end{array}\right)=\frac{n !}{(n-n) ! n !}=1 ; \forall n \in \mathbb{N}
$$

From (5.17) it can be stated that the value of the binomial numbers given in (5.24) can be maintained in extension (5.1) if and only if

$$
\alpha_{1}=1
$$

By gathering the new constraint (5.25) with the previous one (5.15), it is clear that only the sequence corresponding to the subset of the integers, $(i)$, can be considered in all extension of the usual binomials that could preserve its two remarkable values, at least for integer arguments and considering strictly positive integer orders. The sequence of integers can be obtained as a particular case of (5.15) by making $x=0$. 


\begin{tabular}{l|l|l}
${ }_{0} \mathrm{C}_{(i)} 0$ & $i{ }_{+}^{-1} i=1$ & $1(-0)+1(+1)=1$ \\
\hline${ }_{-1} \mathrm{C}_{(i)-1}$ & $i_{+}^{-2}{ }^{i=1}$ & $1(+(-1))+2(-0)+1(+1)=0$ \\
\hline${ }_{-2} \mathrm{C}_{(i)-2}$ & $i_{+}^{-3}{ }^{i=1}$ & $1(-(-2))+3(+(-1))+3(-0)+1(+1)=0$ \\
\hline${ }_{-3} \mathrm{C}_{(i)-3}$ & $i_{+}^{-4}{ }^{i=1}$ & $1(+(-3))+4(-(-2))+6(+(-1))+4(-0)+1(+1)=0$ \\
\hline${ }_{-4} \mathrm{C}_{(i)-4}$ & $i_{+}^{-5}+i=1$ & $1(-(-4))+5(+(-3))+10(-(-2))+10(+(-1))+5(-0)+1(+1)=0$
\end{tabular}

Figure 5: Examples of values of ${ }_{n} \mathrm{C}_{(i) n}$ for $n=0$ and negative orders, obtained by applying the expressions presented in Figure 1.

Given the above, it is interesting to determine if the results of ${ }_{0} \mathrm{C}(i)_{0}$ and

$$
{ }_{n} \mathrm{C}(i)_{n}=\left(\begin{array}{l}
n \\
n
\end{array}\right)_{i}=i_{+}^{n-1} i=1, \forall n \in \mathbb{Z}^{-}
$$

agree with the remarkable value given in the (5.24) extension, for both the null order and the other negative ones. Some results can be found in Figure 5.

From the direct observation of the results, by the property known as Increasing Alternating Sum of Binomial Coefficients (as listed in [1], Chapter 3), it is possible to state that

$$
{ }_{n} \mathrm{C}(i)_{n}=\left(\begin{array}{l}
n \\
n
\end{array}\right)_{i}=0 ; \forall n \in \mathbb{Z}^{-}
$$

Since (5.16) and (5.17) had to be restricted to only the sequence of integers, the only possibility to extend the result of (5.24) to negative integers remains to be the sequence $(i)$. However, as can be seen from (5.27), rather than one, there is an infinity of null results for negative arguments. So it is concluded that

Property 5. There is no sequence of real numbers such that the $T_{S z}$ transformations simultaneously equal to one for binomial numbers $z$ choose zero and $z$ choose $z$, for all integer $z$ values.

\section{ALTERNATIVE FORMS FOR EXTENSION OF THE USUAL BINOMIAL NUMBERS APPLYING TRANSFORMATIONS OF SEQUENCE BY THE SUCCESSIVE SUM}

Considering that there is no sequences of real numbers such that their $T_{\mathrm{Sz}}$ transformations are capable of guaranteeing the unit value of binomials $z$ choose zero and $z$ choose $z$ for every integer, it is valid to inquire whether there is an alternative solution capable of ensuring the validity of these properties for all integers, using $T_{\mathrm{Sz}}$ transformations on more than one sequence. According to this possibility, the following topics propose alternative solutions. 


\subsection{Properties of Interest for Binomial Numbers Extensions}

The procedure for any extension can be started by choosing which properties of the original domain should remain valid for all elements, or most of them, in the new one. In this section, the objective is to extend the validity of five well-known properties of the binomial numbers, from the natural to the integer domain. The selected properties will be the Stifel's relation, the unit value of binomial numbers $n$ choose zero and $n$ choose $n$, the symmetric binomial numbers and the recursive calculus on $k$. Thus, the following expressions and domains are explicitly established: 1 - Stifel's Relation:

$$
{ }_{z+1} \mathrm{C}^{\varepsilon}{ }_{k+1}={ }_{z} \mathrm{C}^{\varepsilon}{ }_{k+1}+{ }_{z} \mathrm{C}^{\varepsilon}{ }_{k} \text {. Best Domain: } \forall k \text { and } z \in \mathbb{Z} .
$$

2 - Binomial Numbers $z$ Choose Zero:

$$
{ }_{z} \mathrm{C}^{\varepsilon}{ }_{0}=1 \text {. Best Domain }: \forall z \in \mathbb{Z} .
$$

3 - Binomial Numbers $z$ Choose $z$ :

$$
{ }_{z} \mathrm{C}^{\varepsilon}{ }_{z}=1 \text {. Best Domain }: \forall z \in \mathbb{Z} \text {. }
$$

4 - Symmetric Binomial Numbers:

$$
{ }_{z} \mathrm{C}^{\varepsilon}{ }_{z}={ }_{z} \mathrm{C}^{\varepsilon}{ }_{z-k} \text {. Best Domain: } \forall k \text { and } z \in \mathbb{Z} \text {. }
$$

5 - Binomial Number $z$ Choose $k+1$, Starting From the Binomial $z$ Choose $k$ :

$$
{ }_{z} \mathrm{C}^{\varepsilon}{ }_{k+1}={ }_{z} \mathrm{C}^{\varepsilon}{ }_{k}(z-k) /(k+1) \text {. Best Domain: } \forall k \neq-1 \text { and } z \in \mathbb{Z} \text {. }
$$

The upper index $\varepsilon$ in $\mathrm{C}$ represents any extension of the usual binomial numbers and not necessarily transformations $T_{\mathrm{Sz}}$ on some sequence $\left(\alpha_{i}\right)$.

Considering this set of five properties, the extension produced from the transformations $T_{\mathrm{Sz}}$ on the sequence of integer numbers $(i)$, as defined in (4.7), presents, respectively, the following results:

$$
\begin{gathered}
{ }_{z+1} \mathrm{C}(i)_{k+1}={ }_{z} \mathrm{C}(i)_{k+1}+{ }_{z} \mathrm{C}(i)_{k} ; \forall k \text { and } z \in \mathbb{Z} . \\
{ }_{z} \mathrm{C}(i)_{0}=1 ; \forall z \in \mathbb{Z} . \\
{ }_{z} \mathrm{C}(i)_{z}=1 ; \forall z \in \mathbb{Z}^{+} . \\
{ }_{z} \mathrm{C}(i)_{z}={ }_{z} \mathrm{C}(i)_{z-k} ; \forall k \in \mathbb{Z}, \text { if } z \in \mathbb{Z}^{+} ; \forall k \in \mathbb{Z}^{-}, \text {if } k-z>0 \text { and } z<-1 . \\
{ }_{z} \mathrm{C}(i)_{k+1}={ }_{z} \mathrm{C}(i)_{k}(z-k) /(k+1) ; \forall k \neq-1 \text { and } z \in \mathbb{Z} .
\end{gathered}
$$

Details about the determination of the domains in (6.6)-(6.10) can be found in [9].

When comparing the domains of (6.6)-(6.10) with those of (6.1)-(6.5) it is possible to conclude that ${ }_{z} \mathrm{C}(i)_{k}$ is an extension that serves well in most situations that consider the properties of 
interest, except if it is important to obtain the best domain possible to binomial numbers $z$ choose $z$, if $z$ is negative, or to keep the symmetry for all extended values.

In [9] a quantitative evaluation methodology has also been defined which is applicable to all function extensions from natural arguments to integer ones. That proposition allows to associate an objective global index for each binomial numbers extension, which can be useful if it is necessary to choose one extension among several others that do not fit the best domain established according to some properties of interest. In what follows, it will be presented a solution for the above specified set of properties, according to this methodology.

\subsection{Binomial Extension Applying the $p$ and $n$ Component Transformations of the Integer Numbers Sequence}

A sequence may be constructed by proper operations on the terms of other ones. In fact, this property has already been used in Section 2 for the $T_{\mathrm{Sz}}$ transform definition. For example, let $G(A,+)$ be a group and $S Z$ the integer sequences set over $A$. Under these conditions, consider two sequences on $A$ :

$$
\begin{aligned}
\left(\alpha_{i}\right)_{i \in \mathbb{Z}} & =\left(\ldots, \alpha_{-1}, \alpha_{0}, \alpha_{1}, \alpha_{2}, \ldots, \alpha_{i}, \ldots\right) . \\
\left(\beta_{i}\right)_{i \in \mathbb{Z}} & =\left(\ldots, \beta_{-1}, \beta_{0}, \beta_{1}, \beta_{2}, \ldots, \beta_{i}, \ldots\right) .
\end{aligned}
$$

It will always be possible to construct a new sequence in the form

$$
\left(\alpha_{i}+\beta_{i}\right)_{i \in \mathbb{Z}}=\left(\ldots, \alpha_{-1}+\beta_{-1}, \alpha_{0}+\beta_{0}, \alpha_{1}+\beta_{1}, \alpha_{2}+\beta_{2}, \ldots, \alpha_{i}+\beta_{i}, \ldots\right) .
$$

Under such conditions, it is established that $\left(\alpha_{i}\right)$ and $\left(\beta_{i}\right)$ are components of $\left(\alpha_{i}+\beta_{i}\right)$, and the latter is their composition by the +operator.

In this section, two integer sequence components will be operated by the transformations $T_{\mathrm{Sz}}$, and the corresponding results will be used in a binomial numbers extension that aims the better domain of the properties of interest, established in Section 6.1. Thus, the sequences $\left(p_{i}\right)$ and $\left(n_{i}\right)$ are defined:

$$
\begin{aligned}
& \left(p_{i}\right)_{i \in \mathbb{Z}}= \begin{cases}(i)_{i \in \mathbb{Z}} ; & \text { if } i \geq 0 \\
(0)_{i \in \mathbb{Z}} ; & \text { if } i<0\end{cases} \\
& \left(n_{i}\right)_{i \in \mathbb{Z}}= \begin{cases}(0)_{i \in \mathbb{Z}} ; & \text { if } i \geq 0 \\
(i)_{i \in \mathbb{Z}} ; & \text { if } i<0\end{cases}
\end{aligned}
$$

The sequences $\left(p_{i}\right)_{i \in \mathbb{Z}}$ and $\left(n_{i}\right)_{i \in \mathbb{Z}}$ are called components $p$ and $n$ of $(i)_{i \in \mathbb{Z}}$, since it is immediate to conclude that

$$
\left(p_{i}+n_{i}\right)_{i \in \mathbb{Z}}=(\ldots,-2,-1,0,1,2, \ldots, i, \ldots)=(i)_{i \in \mathbb{Z}}
$$

The addition of the successive sum of any two sequences is equal to the successive sum of the composition by the addition of these sequences (see appendix B of [9]):

$$
\alpha_{i+}^{\mid i=z}+\beta_{i_{+}^{\mid}}^{i=z}=\left(\alpha_{i}+\beta_{i}\right)_{+}^{\mid i=z} ; \forall z \in \mathbb{Z} .
$$


The result in (6.17) can be extended without further difficulties to other transformations by the successive sum. Then, from (6.14)-(6.17) it can be inferred that

$$
p_{i}{ }_{+}^{k} \boldsymbol{i}=z+n_{i}{ }_{\dot{+}}^{k} \boldsymbol{i}=z=\left(p_{i}+n_{i}\right)_{\dot{+}}^{k} i=z=i_{\dot{+}}^{k} i=z ; \forall k \text { and } z \in \mathbb{Z} \text {. }
$$

Although (6.18) may suggest that it is indifferent to apply $T_{\mathrm{Sz}}$ on the components or on their composition, those transformations by the successive sum considered individually on the sequences $\left(p_{i}\right)$ and $\left(n_{i}\right)$ result in distinct sequences of the transform applied on $\left(p_{i}+n_{i}\right)$.

The difference between the results occurs due to the symmetric values cancellation when the component transformations are added, producing several null results in the composition. Taking advantage of this difference a definition of binomial numbers extension was proposed in [9] using two sentences, as follows.

\section{Binomial Extension 2.}

Let $G(Z,+)$ be a group, $\left(p_{i}\right)$ and $\left(n_{i}\right)$ sequences in $S Z$ set, defined by the expressions (6.14) and (6.15) respectively. Under these conditions, it will be defined the binomial number " $z$ choose $k$ in components $l$ ", or the combinatorial number " $z$ choose $k$ in components $l$ ", through the expressions

$$
{ }_{z} \mathrm{C}^{\imath}{ }_{k}=\left\{\begin{array}{l}
{ }_{z} \mathrm{C}\left(p_{i}\right)_{k}=p_{i}{ }^{k-1}+i=z-k+1, \text { if }(z-k) \geqslant 0, \\
{ }_{z} \mathrm{C}\left(n_{i}\right)_{k}=n_{i}{ }^{k-1}+i=z-k+1 ; \text { if }(z-k)<0 ; k \text { and } z \in \mathbb{Z} .
\end{array}\right.
$$

Remark: the exponent " $l$ " is not an assignable index but rather the extension identification. As exponent of $\mathrm{C}$, it is reserved in this article only for the extension of the binomial numbers through the components $p$ and $n$ of $(i)$.

To make compatible the notation applied in the definition (6.19)-(6.20) with the classical binomial number representation, it follows that

$$
{ }_{z} \mathrm{C}_{k}{ }_{k}=\left(\begin{array}{l}
z \\
k
\end{array}\right)_{l} ; \forall k \text { and } z \in \mathbb{Z}
$$

Figure 6 exemplifies some of the binomial values in $t$.

Considering the same set of five properties established in Section 6.1, the composite extension, elaborated from the $T_{\mathrm{Sz}}$ transformations on the components $\left(p_{i}\right)$ and $\left(n_{i}\right)$ of the integer's sequence (i), yields the following results:

$$
\begin{gathered}
{ }_{z+1} \mathrm{C}^{l}{ }_{k+1}={ }_{z} \mathrm{C}_{k+1}^{l}+{ }_{z} \mathrm{C}_{k}^{l} ; \forall k \neq-1 \text { and } z \neq-1 \in \mathbb{Z} . \\
{ }_{z} \mathrm{C}^{l}{ }_{0}=1 ; \forall z \in \mathbb{Z} . \\
{ }_{z} \mathrm{C}^{l}{ }_{z}=1 ; \forall z \in \mathbb{Z} . \\
{ }_{z} \mathrm{C}^{l}{ }_{z}={ }_{z} \mathrm{C}^{l}{ }_{z-k} ; \forall k \text { and } z \in \mathbb{Z} .
\end{gathered}
$$




\begin{tabular}{|c|c|c|c|c|c|c|c|c|c|}
\hline$i i^{-4} i=-1$ & 0 & $\left(\begin{array}{l}-5 \\
-2\end{array}\right)_{\imath}$ & $n_{i} \stackrel{-3}{i} i=-2^{-1}$ & 0 & $\left(\begin{array}{l}-5 \\
-1\end{array}\right)_{2}$ & $n_{i} \stackrel{-2}{+} i=-3^{-1}$ & 0 & $\left(\begin{array}{r}-5 \\
0\end{array}\right)_{\imath}$ & $n_{i} \stackrel{-1}{+}^{i=-4}$ \\
\hline 0 & $\left(\begin{array}{l}-4 \\
-2\end{array}\right)_{2}$ & $n_{i} \stackrel{-3}{+} i=-1^{-1}$ & 0 & $\left(\begin{array}{l}-4 \\
-1\end{array}\right)_{\imath}$ & $n_{i} \stackrel{-2}{+} i=-2^{-2}$ & First Ex & xtension & $l_{i} \ddot{+}^{-1} i=-3$ & 1 \\
\hline Secc & nd Exte & nsion Ne & w Values & $i^{-2} i=-1$ & 0 & $\left(\begin{array}{r}-3 \\
0\end{array}\right)_{\imath}$ & $n_{i} \stackrel{-1}{+}^{i=-2}$ & 1 & $\left(\begin{array}{r}-3 \\
1\end{array}\right)_{\imath}$ \\
\hline$i+^{-3} i=1$ & 1. & $\left(\begin{array}{l}-2 \\
-1\end{array}\right)_{\imath}$ & $n_{i} i^{-2} i=0$ & 0 & $\left(\begin{array}{r}-2 \\
0\end{array}\right)_{\imath}$ & $n_{i} \ddot{+}^{-1} i=-1$ & 1 & $\left(\begin{array}{r}-2 \\
1\end{array}\right)_{\imath}$ & $n_{i} \stackrel{0}{i} i=-2$ \\
\hline-1 & $(-$ & $p_{i}^{-2} i=1$ & 1 & $\left(\begin{array}{r}-1 \\
0\end{array}\right)_{\imath}$ & $n_{i} \stackrel{-1}{+}^{i=0}$ & 1 & $\left(\begin{array}{r}-1 \\
1\end{array}\right)_{\imath}$ & $n_{i} \stackrel{\circ}{+}^{i=-1}$ & -1 \\
\hline $\left.\begin{array}{r}0 \\
-1\end{array}\right)$ & $p_{i}{ }^{-2} i=2$ & 0 & $\left(\begin{array}{l}0 \\
0\end{array}\right)_{\imath}$ & $p_{i} \stackrel{-1}{+} i=1^{-1}$ & 1 & $\left(\begin{array}{l}0 \\
1\end{array}\right)_{\imath}$ & $n_{i} \dot{\circ}_{+}^{i=0}$ & 0 & $\left(\begin{array}{l}0 \\
2\end{array}\right)_{\imath}$ \\
\hline$b_{i}^{-2} i=3$ & 0 & $\left(\begin{array}{l}1 \\
0\end{array}\right)_{\imath}$ & $p_{i} \dot{+}^{i} i=2$ & 1 & $\int_{\imath}$ & $p_{i} \stackrel{0}{+}^{i=1}$ & 1 & $\underline{2}$. & $n_{i} \dot{i}+i=0$ \\
\hline 0 & $\left(\begin{array}{l}2 \\
0\end{array}\right)_{\imath}$ & $p_{i} \dot{+}^{-1} i=3$ & 1 & $\left(\begin{array}{l}2 \\
1\end{array}\right)_{\imath}$ & $p_{i} \stackrel{\circ}{+}^{i=2}$ & 2 & $\left(\begin{array}{l}2 \\
2\end{array}\right)_{\imath}$ & $p_{i}{ }^{i=1}$ & 1 \\
\hline$\left(\begin{array}{l}3 \\
0\end{array}\right)_{\imath}$ & $p_{i} \dot{+}^{i=4}$ & 1 & Origin & nal Binor & mial Nun & mbers & $p_{i}+i=2$ & 3 & $\left(\begin{array}{l}3 \\
3\end{array}\right)_{\imath}$ \\
\hline$p_{i} \dot{-1}^{i}=5$ & 1 & $\left(\begin{array}{l}4 \\
1\end{array}\right)_{\imath}$ & $p_{i} \stackrel{i}{i}^{i=4}$ & 4 & $\left(\begin{array}{l}4 \\
2\end{array}\right)_{\imath}$ & $p_{i}+{ }^{i=3}$ & 6 & $\left(\begin{array}{l}4 \\
3\end{array}\right)_{\imath}$ & $p_{i} \stackrel{2}{+}^{i=2}$ \\
\hline 1 & $\left(\begin{array}{l}5 \\
1\end{array}\right)_{\imath}$ & $p_{i} \stackrel{0}{+}^{i=5}$ & 5 & $\left(\begin{array}{l}5 \\
2\end{array}\right)_{\imath}$ & $p_{i} \dot{1}^{i=4}$ & 10 & $\left(\begin{array}{l}5 \\
3\end{array}\right)_{\imath}$ & $p_{i} \stackrel{2}{+}^{i=3}$ & 10 \\
\hline
\end{tabular}

Figure 6: Extension results of binomial numbers in $\imath$. The thicker dashed line separates the values obtained by the component $\left(p_{i}\right)$ from those that are computed using $\left(n_{i}\right)$, and the region containing values highlighted in white on a dark background is not necessarily null, differently from the first extension. More results can be found in [9].

$$
{ }_{z} \mathrm{C}^{l}{ }_{k+1}={ }_{z} \mathrm{C}^{l}{ }_{k}(z-k) /(k+1) ; \forall k \neq-1 \text { and } z \in \mathbb{Z} .
$$

The domains verification in (6.22)-(6.26) can be found in [9].

By comparing the domains, it can be seen that the binomial numbers extension through components transformations on sequences indexed by integers maintains the same validity domain of the first extension and also eliminates the constraints of ${ }_{z} \mathrm{C}_{(i) k}$ in the case of binomial numbers $z$ choose $z$ and the symmetric binomials. So the validity domains of these properties are extended to the best possible domain. In contrast, the Stifel relation becomes invalid in the case $z=k=-1$, as can be seen from the set of the three central values of Figure 6:

$$
\left(\begin{array}{l}
0 \\
0
\end{array}\right)_{l} \neq\left(\begin{array}{r}
-1 \\
0
\end{array}\right)_{l}+\left(\begin{array}{l}
-1 \\
-1
\end{array}\right)_{l}
$$

Although this is the only one discordant value, the remaining question is whether this is tolerable or not, which must be solved by analyzing the specific application intended. In cases where it is preferable to guarantee the relation of Stifel for all integer arguments instead of the unit value for the binomial numbers ${ }_{z} \mathrm{C}_{0}^{l}$ and ${ }_{z} \mathrm{C}_{z}$, it will be more appropriate to use another extension, such as the one presented in Section 4. 


\subsection{Compact Representation for Binomial Numbers Through Component Transforma- tions $p$ and $n$ of the Integer's Sequence}

Comparing the values in Figure 6 and Figure 4, it is verified that a large part of the ${ }_{z} \mathrm{C}_{k}{ }_{k}$ values is equal to others of the binomial number's first extension:

$$
{ }_{z} \mathrm{C}^{l}{ }_{k}=(z-k+1) \stackrel{k-1}{+} ; \forall k \geqslant 0 \text { and } z \in \mathbb{Z} .
$$

This type of representation is more simple than that which uses the sequence components and has the additional advantage of involving only successive sums on the integers themselves. These are supposedly known results, for example, from the first extension, so they do not need to be recalculated. It is interesting to conjecture if a similar representation can be achieved for the other results of this extension, that is, for integer arguments in $z$ and negative integers in $k$. This conjecture has been proved true in [9], during an investigation of the domain of validity of the symmetry property in the extension ${ }_{z} \mathrm{C}_{k}$. Part of the results obtained is shown in Figure 7.

\begin{tabular}{|c|c|}
\hline$k$ & Symmetrical Binomials ${ }_{z} \mathrm{C}_{z-k}={ }_{z} \mathrm{C}_{k}{ }_{k}$ For Negative Values of $k$ \\
\hline$k=-1$ & ${ }_{z} \mathrm{C}_{z-1}^{\imath}={ }_{z} \mathrm{C}_{-1}^{\imath}=0_{+}^{\mathrm{z}}=\left\{\begin{array}{r}+1, \text { if } z=-1, \text { and } \\
0 \text {; if } z \neq-1 .\end{array}\right.$ \\
\hline$k=-2$ & $=\left\{\begin{aligned}+1, & \text { if } z=-2 \\
-1, & \text { if } z=-1, \text { and } \\
0 & \text {; in other cases. }\end{aligned}\right.$ \\
\hline$k=-3$ & ${ }_{z} \mathrm{C}_{z-3}{ }_{z-} \mathrm{C}_{-3}^{\imath}=(-2)_{+}^{z+2}=\left\{\begin{aligned}+1, & \text { if } z=-3, \\
-2, & \text { if } z=-2, \\
+1, & \text { if } z=-1, \text { and } \\
0 & \text { in other cases. }\end{aligned}\right.$ \\
\hline$\ldots$ & 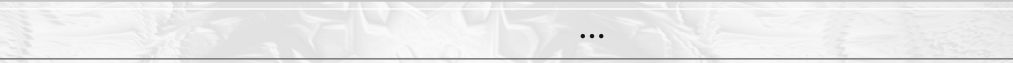 \\
\hline$k$ & ${ }_{z} \mathrm{C}_{z-k}={ }_{z} \mathrm{C}_{k}^{\imath}=(k+1)^{\mathrm{z}-\mathrm{k}-1} ; \forall z$ and $k<0 \in \mathbb{Z}$. \\
\hline
\end{tabular}

Figure 7: For negative $k$ the symmetric binomials of the ${ }_{z} \mathrm{C}^{l}{ }_{k}$ extension have values equal to those obtained through the $T_{\mathrm{Sz}}$ transformations on the sequence of the integers; however, these results produce a generalization different from that obtained for positive $k$. 


\begin{tabular}{|c|c|c|c|c|c|c|c|c|c|}
\hline$-2_{i}^{-3}$ & 0 & $\left(\begin{array}{l}-5 \\
-2\end{array}\right) \imath$ & $-1 t_{+}^{-4}$ & 0 & $\left(\begin{array}{l}-5 \\
-1\end{array}\right)_{2}$ & $0 \stackrel{-5}{+}^{-5}$ & 0 & $\left(\begin{array}{r}-5 \\
0\end{array}\right)_{\imath}$ & $-4 \ddot{t}^{-1}$ \\
\hline 0 & $\left(\begin{array}{l}-4 \\
-2\end{array}\right)_{\imath}$ & $-1 \stackrel{-3}{+}^{-3}$ & 0 & $\left(\begin{array}{l}-4 \\
-1\end{array}\right)_{\imath}$ & $0+4$ & \multicolumn{2}{|c|}{ First Extension } & $-3 \ddot{+}$ & 1 \\
\hline \multicolumn{4}{|c|}{ Second Extension New Values } & $0_{+}^{-3}$ & 0 & $\left(\begin{array}{r}-3 \\
0\end{array}\right)_{\imath}$ & $-2 \ddot{t}^{-1}$ & 1 & $\left(\begin{array}{r}-3 \\
1\end{array}\right)_{\imath}$ \\
\hline$-1 \bar{t}$ & 1 & $\left(\begin{array}{l}-2 \\
-1\end{array}\right)_{2}$ & $0 \stackrel{-2}{+}$ & 0 & $\left(\begin{array}{r}-2 \\
0\end{array}\right)_{\imath}$ & $-1 \hat{t}^{-1}$ & 1 & $\left(\begin{array}{r}-2 \\
1\end{array}\right)_{\imath}$ & $-2 \stackrel{i}{+}$ \\
\hline-1 & $\left(\begin{array}{l}-1 \\
-1\end{array}\right)_{2}$ & $0_{+}^{-1}$ & 1 & $\left(\begin{array}{r}-1 \\
0\end{array}\right)_{2}$ & $0 \ddot{q}$ & 1 & $\left(\begin{array}{r}-1 \\
1\end{array}\right)_{\imath}$ & $-1 \stackrel{0}{+}$ & -1 \\
\hline$\left(\begin{array}{r}0 \\
-1\end{array}\right)_{2}$ & $0 \stackrel{\circ}{+}$ & 0 & $\left(\begin{array}{l}0 \\
0\end{array}\right)_{\imath}$ & $1 \ddot{t}$ & 1 & $\left(\begin{array}{l}0 \\
1\end{array}\right)_{2}$ & $0 \stackrel{i}{+}$ & $\mathbf{0}$ & $\left(\begin{array}{l}0 \\
2\end{array}\right)_{\imath}$ \\
\hline $0+$ & 0 & $\left(\begin{array}{l}1 \\
0\end{array}\right)_{\imath}$ & $2-1$ & 1 & $\left(\begin{array}{l}1 \\
1\end{array}\right)_{\imath}$ & $1 \stackrel{0}{+}$ & 1 & $\left(\begin{array}{l}1 \\
2\end{array}\right)_{2}$ & $0+$ \\
\hline 0 & $\left(\begin{array}{l}2 \\
0\end{array}\right)_{\imath}$ & $3 \ddot{t}$ & 1 & $\left(\begin{array}{l}2 \\
1\end{array}\right)_{\imath}$ & $2 \dot{+}$ & 2 & $\left(\begin{array}{l}2 \\
2\end{array}\right)_{\imath}$ & $1+$ & 1 \\
\hline$\left(\begin{array}{l}3 \\
0\end{array}\right)_{\imath}$ & $4 \ddot{t}$ & 1 & \multicolumn{4}{|c|}{ Original Binomial Numbers } & $2+$ & 3 & $\left(\begin{array}{l}3 \\
3\end{array}\right)_{\imath}$ \\
\hline $5 \ddot{t}^{-1}$ & 1 & $\left(\begin{array}{l}4 \\
1\end{array}\right)_{2}$ & $4 \stackrel{0}{+}$ & 4 & $\left(\begin{array}{l}4 \\
2\end{array}\right)_{2}$ & $3+$ & 6 & $\left(\begin{array}{l}4 \\
3\end{array}\right)_{2}$ & $2 \stackrel{2}{+}$ \\
\hline 1 & $\left(\begin{array}{l}5 \\
1\end{array}\right)_{\imath}$ & $5 \stackrel{i}{+}$ & 5 & $\left(\begin{array}{l}5 \\
2\end{array}\right)_{\imath}$ & $4+$ & 10 & $\left(\begin{array}{l}5 \\
3\end{array}\right)_{\imath}$ & $3 \stackrel{2}{+}$ & 10 \\
\hline
\end{tabular}

Figure 8: Compact representation of ${ }_{z} \mathrm{C}_{k}{ }_{k}$ using the transformations $T_{\mathrm{Sz}}$ applied on the integer's sequence instead of their application on the components $p$ and $n$.

The possibility of representing ${ }_{z} \mathrm{C}_{k}$ in terms of transformations by the successive sum on integer's sequence has been also confirmed in [9], then

$$
{ }_{z} \mathrm{C}^{l}{ }_{k}=\left(\begin{array}{l}
z \\
k
\end{array}\right)_{\imath}=\left\{\begin{array}{l}
(z-k+1)^{k-1}+\text { if }(z-k) \geqslant 0, \\
(k+1)^{z-k-1}+\text { if }(z-k)<0 ; k \text { and } z \in \mathbb{Z} .
\end{array}\right.
$$

From (6.29) and (6.30) this extension has a simple representation like that of the first extension, which can be seen by comparing Figures 8 and 6 .

\subsection{Binomial Numbers Extension Through Gamma Function Symmetries}

The topic of binomial numbers extension is of great interest and the subject is far from being exhausted. Some propositions have been made in the literature to extend the domain from natural to the integer numbers, as in [6], using combinatorial arguments, and as in [7] and [12], dealing only with the extension of the usual factorials; those extensions also lead to binomial numbers extensions. To substantiate this importance, it will be detailed the following case. The binomial numbers can be defined generically for complex arguments, from the gamma function, [1], by

$$
{ }_{x} \mathrm{C}_{y}=\Gamma(x+1) /[\Gamma(y+1) \Gamma(x-y+1)] ; x \text { and } y \in \mathbb{C} .
$$


which produces the same results that can be obtained by expression (4.1) of ${ }_{n} \mathrm{C}_{k}$ when $x$ and $y$ are the natural numbers $n$ and $k$ respectively. Although this is an important extension, the question of obtaining binomial numbers for $x$ and $y$ negative integers can not be solved by applying (6.31), because the gamma function is not defined in $\mathbb{Z}, \mathbb{Q}, \mathbb{R}$ or $\mathbb{C}$ for those values.

In 2015 Kronenburg [8] proposed the extension ${ }_{z} \mathrm{C}^{\kappa}{ }_{k}$ of binomial numbers for any arguments in $\mathbb{Z}$, based on the symmetry properties of the gamma function:

1. For $z$ positive integer and $k$ any integer:

$$
{ }_{z} \mathrm{C}^{\kappa}{ }_{k}=\left(\begin{array}{l}
z \\
k
\end{array}\right)=\left\{\begin{array}{cc}
\frac{z !}{(z-k) ! k !} ;(z-k) \geqslant 0 ; \forall k \text { and } z \in \mathbb{Z}^{+} . \\
0 ; \quad \text { in other cases. }
\end{array}\right.
$$

2. For $z$ negative integer and $k$ any integer:

$$
{ }_{z} \mathrm{C}^{\kappa}{ }_{k}=\left(\begin{array}{l}
z \\
k
\end{array}\right)=\left\{\begin{array}{cc}
(-1)^{k}\left(\begin{array}{c}
-z+k-1 \\
k
\end{array}\right) & ; \text { if } k \geqslant 0 ; \forall z \in \mathbb{Z}^{-} . \\
(-1)^{z-k}\left(\begin{array}{c}
-k-1 \\
z-k
\end{array}\right) ; \text { if } k \leqslant z ; \forall z \in \mathbb{Z}^{-} . & \text {in other cases } .
\end{array}\right.
$$

By comparison, it can be concluded that the results generated by (6.29)-(6.30), presented in Figure 8, are equal to the results obtained by (6.32)-(6.36). However, it is possible to perceive the following advantages when choosing to define and extend the binomial numbers through the transformations $T_{\mathrm{Sz}}$ :

1. the methodology using $\mathrm{T}_{\mathrm{Sz}}$ transformations presented here avoid any use of complex numbers to make binomial numbers extensions;

2. the definitions using $\mathrm{T}_{\mathrm{Sz}}$ transformations require less amount of sentences than other methodologies;

3. application of additions and subtractions instead of multiplications and divisions;

4. transformations by successive sum allow establishing easily an infinity of binomial numbers and their extensions preserving the properties of interest for specific applications.

\section{CONCLUSIONS}

This paper presented a more general way to define binomial numbers through additions rather than multiplications. The proposed alternative method made possible to extend the domain of application of other binomials in the form ${ }_{z} \mathrm{C}^{\varepsilon}$ for all integer arguments.

By introducing the sequence transformations through the successive sum it was possible to establish binomial numbers through simple additions, dismissing multiplications and divisions of 
factorials, what made also possible to consider negative integer indexes as arguments without difficulties.

The present methodology proposed an additional criterion for choosing the most appropriate extension, since there is an infinity of possible results that could be obtained. Therefore, when specifying the properties of interest, a filter was created to eliminate solutions until that it remains only the ones that matter.

When it is not possible to satisfy some properties of interest for all the domain, it is already possible to employ more than one transformation by the successive sum. In this sense, the definition of binomial numbers applying transformations by the successive sum on the integer's sequence components was applied with success. Other noticeable points regarding this extension are the fact that, once more, the results are obtained simply by usual additions, differently from other techniques available in the current literature, and it was possible to extend them to any integer argument.

RESUMO. A definição clássica de números binomiais envolve fatoriais, tornando inviável sua extensão para inteiros negativos. A metodologia empregada neste artigo permite estabelecer diversas novas extensões de números binomiais para domínios inteiros, reproduz para argumentos inteiros extensões propostas em outros trabalhos e verifica, também, os resultados dos números binomiais clássicos. Para isto, a impossibilidade de computar fatoriais com argumentos inteiros negativos é eliminada pela substituição da definição clássica dos binomiais por uma nova definição com base em operações recentemente propostas e, até o momento, referidas como transformações pela soma sucessiva aplicada em sequências indexadas por inteiros. Particularizando tais operações para sequências inteiras formadas pelos próprios números inteiros, é possível redefinir os números binomiais usuais para qualquer argumento inteiro, com a vantagem de que os valores são mais facilmente computados pelo uso de adições em vez de multiplicações, divisões ou outras combinações mais elaboradas dessas operações, que poderiam requerer mais do que uma ou duas sentenças em suas aplicações.

Palavras-chave: matemática discreta, estruturas algébricas, recursões, sequências, produto sucessivo, soma sucessiva, números binomiais.

\section{REFERENCES}

[1] M. Abramowitz \& I.A. Stegun. "Handbook of Mathematical Functions with Formulas, Graphs and Mathematical Tables". National Bureal of Standarts Applied Mathematics Series, 55. U.S. Government Printing Office, Washington D.C. (1972), 256-265 pp.

[2] S. Aljohani. History of Bionomial Theory. International Journal of Scientific and Engineering Research, 7 (2016), 161-162.

[3] S. Butler \& P. Karasik. A Note on Nested Sums. Journal of Integer Sequences-Article 10.4.4, 13 (2010). 
[4] L.D. Ferreira. Integer Binomial Plan: a Generalization on Factorials and Binomial Coefficients. Journal of Mathematics Research, 2 (2010), 18-35.

[5] D. Goss. "Arithmetic Geometry over Global Function Fields". Advanced Courses in Mathematics. Birkhauser, Basel, Barcelona (2014), 183-193 pp. URL https://link. springer . com/book/10. 1007/978-3-0348-0853-8. See chapter "Ongoing Binomial Revolution".

[6] R.L. Graham, D.E. Knuth \& O. Patashnik. "Concrete Mathematics". Addison-Wesley Publishing Company, Massachusetts, 2 ed. (1994), 153-204 pp.

[7] A.M. Ibrahim. Extension of factorial concept to negative numbers. Notes on Number Theory and Discrete Mathematics, 19 (2013), 30-42.

[8] M. Kronenburg. The Binomial Coefficient for Negative Arguments. arXiv:1105.3689v2 [math.CO], (2015), 1-9.

[9] M. Moesia B. Uma nova metodologia para a extensão de domínio de operações matemáticas sucessivas, com aplicações na análise combinatória. Master Thesis, Laboratório Nacional de Computação Científica, LNCC (2017).

[10] M. Moesia B., J. Karam F. \& G. A. Giraldi. Uma nova metodologia para a extensão de domínio de operações matemáticas sucessivas. In "Proceeding Series of the Brazilian Society of Computational and Applied Mathematics", volume 6. SBMAC (2018). URL https ://proceedings . sbmac . org. $\mathrm{br} / \mathrm{sbmac} / \mathrm{article} / \mathrm{view} / 2544 / 2563$.

[11] Philip J. Davis. Leonhard Euler's Integral: A Historical Profile of the Gamma Function: In Memoriam: Milton Abramowitz. The American Mathematical Monthly, 66 (1959), 849-869.

[12] A.K. Thukral. Factorials of real negative and imaginary numbers - A new perspective. Springer Plus, 3:658 (2014). URL http: //www . springerplus . com/content/3/1/658. 